\title{
The Availability of Resources for Standard Management of Hypertension at Public Primary Health Care Facilities, Shargelneel Locality-Khartoum State-Sudan
}

\author{
Naiema Abdalla Wagialla ${ }^{1}$, Mustafa Khidir Mustafa Elnimeiri ${ }^{2}$ \\ ${ }^{1}$ Community Medicine, Non-communicable Disease Directorate, Federal Ministry of Health, Khartoum, Sudan \\ ${ }^{2}$ Faculty of Medicine, Department of Community Medicine, Elneelain University, Khartoum, Sudan
}

Email address:

naiemafahal@gmail.com (N. A. Wagialla),naiemafahal@gmail.com (M. K. M. Elnimeiri)

\section{To cite this article:}

Naiema Abdalla Wagialla, Mustafa Khidir Mustafa Elnimeiri. The Availability of Resources for Standard Management of Hypertension at Public Primary Health Care Facilities, Shargelneel Locality-Khartoum State-Sudan. Journal of Family Medicine and Health Care.

Vol. 2, No. 4, 2016, pp. 138-141. doi: 10.11648/j.jfmhc.20160204.27

Received: October 26, 2016; Accepted: November 24, 2016; Published: January 12, 2017

\begin{abstract}
Introduction: Hypertension is already a high prevalent risk factor for Cardio Vascular Diseases (CVDs) throughout the world due to prevalence of contributing modifiable risk factors such as unhealthy diet, physical inactivity, tobacco use, and Hyperlipidemia. Worldwide hypertension estimated to cause 7.1 million premature death and $4.5 \%$ of disease burden (64 million disability adjusted life years (DALYs), it is eminently preventable, and this can be achieved by targeted life-related risk factors. Objective: To study the availability of resources needed for standard management of hypertension in Public Primary Health Care Facilities, in Sharg-Alneel locality, Khartoum State, aiming to generate information for establishing quality control program. Methodology: Facility- based cross-sectional descriptive study was conducted at Sharg-Alneel locality, Khartoum State, Sudan. The study Population composed of 26 public primary health care facilities and 3 rural hospitals, and all health care providers available during the data collection period and they were 119 care providers. Standardized administered questionnaire and checklist were developed, pre-test and used for data collection. The data was analyzed using the Statistical Package for Social Science (SPSS) version 15. Results: Marked shortage in care providers (physicians and nurses), only 0.5-physician per10,000 populations, and 0.6 nurse per10,000 populations. In addition, $71.4 \%$ of the physician and $93.5 \%$ of other health care providers were not subjected to training on standard management of hypertension. Standard referral, reporting and recording systems, were not established yet, as well, hypertension health education materials and national hypertension guidelines were not available in all health facilities. Sphygmomanometer, adult weighing scale, ophthalmoscope, x-ray machine and ECG machines, were available in $96.6 \%$, $93.1 \%, 24.2 \%, 20.7 \%, 17.2 \%$ of the health facilities respectively. In addition, essential investigations e.g. reagent for cholesterol, blood creatinine \& blood urea kits, and reagent for uric acid test, were available in $20.7 \%, 31 \%, 62.1 \%$, and $37.9 \%$ of the health facilities respectively. Only three out of 13 anti-hypertensive drugs included in the national drug list of primary health care level were available in the health facilities, and these were; Aspirin (86\%), Furosemide (62\%) and Atenolol (52\%). Conclusion: There was a marked shortage in health care providers, only 0.5-physician per 10,000 populations, and 0.6 nurses per 10, 000 populations. Other resources were inadequate to provide quality services for hypertensive patients at the primary health care level.
\end{abstract}

Keywords: Care Providers, Training, Equipment, Investigations, Anti-hypertensive Drugs, National Guideline, Ambulance, Primary Health Care Facilities

\section{Introduction}

Hypertension is already a high prevalent risk factor for cardio-vascular diseases (CVDs) throughout the world due to prevalence of contributing modifiable risk factors such as unhealthy diet, physical inactivity, tobacco use, and hyperlipidemia. It is the third leading killer in the world. There are one billion hypertensive patients globally. 
Worldwide hypertension estimated to cause 7.1 million premature death and $4.5 \%$ of disease burden (64 million disability adjusted life years (DALYs). In Eastern Mediterranean Region, the prevalence of hypertension averages $26 \%$ and it affects approximately 125 million individuals (1). In Sudan, hypertension has the highest prevalence among the major NCDs (24.3\%) (2). Hypertension represented as one of the 10 leading diseases treated in health facilities, and one of the 10 leading causes of deaths in Sudan (3). However, it is eminently preventable, and this can be achieved by targeted life-related risk factors. Treating hypertension has been associated with about a $40 \%$ reduction in the risk of stroke and about a $15 \%$ reduction in the risk of myocardial infarction, so with standard management of hypertension, we can reduce the major complications that lead to morbidity, disability and mortality (4).

Of an estimated 58 million deaths globally from all causes in 2005, cardiovascular disease (CVD) accounted for $30 \%$ (5). It is important to recognize that a substantial proportion of these deaths (46\%) were of people under 70 years of age, in the more productive period of life; in addition, $79 \%$ of the disease burden attributed to cardiovascular disease is in this age group (6). It is already place a significant economic burden on low \& middle-income countries (Sudan one of these countries) (7), in spite of this, the resources available for its management in these countries are limited because of competing health priorities. Nevertheless, it is essential to recognize that the transition to lower levels of infectious diseases and higher levels of non-communicable diseases is already under way; failure to act now will result in large increases in avoidable CVDs, placing serious pressures on the national economies (8-10). One of the major tasks for WHO and its Member States are to scale up cost-effective, integrated approaches for prevention of CVDs as included in Global Strategy (11)

\subsection{Justification}

- Hypertension is not symptomatic until late in the development of the disease, so due to lack of appropriate prevention, early detection and care, many people are unnecessarily suffering from hypertensionand its complications, such as coronary heart diseases, strokes, chronic renal failure and blindness.

- It is necessary that standard management of hypertension is to be available at the primary health care (PHC) level, where high percentage of population use PHC, as it is more accessible and affordable and hence it has a drive to reach vulnerable populations. Added to this, in $\mathrm{PHC}$, health promotion, prevention and care services can be provided at the same place.

- In Sudan, delivery of care has been based on the primary health care (PHC) approach, and as stated in
Sudan Health Map 2012, there were 2040 PHC facilities, supposed to delivere the above mentioned services, but have never functioned as such.

Thus, it is justifiable to conduct the study in order to highlight the availability of resources needed for standard management of hypertension and its effect on the currently provided services at the PHC facilities.

\subsection{Objective}

The overall objective: To study the availability of resources needed for standard management of hypertension in Public Primary Health Care Facilities, in Sharg-Alneel locality, Khartoum State, Sudan aiming to generate information for establishing quality control program.

\section{Methodology}

Facility- based cross-sectional descriptive study was conducted including 26 public health centres and 3 rural hospitals in Sharg-Alneel locality, Khartoum State. The study population was composed of all the health care providers in the public health facilities available during the data collection period after their consent had been obtained. They were 119 care providers, included; Physicians and other care providers, of various categories Three tools for data collection were used:

(1) A self-administered questionnaire for the physicians, included 33 questions (2) Administered questionnaire for the other care providers, included 29 questions (3) and Standard checklist for the facilities, involved 40 items. All questionnaires were close-ended. An ethical approval was obtained from The Sudan Medical Specialization Board. A verbal consent was obtained from each participant. The collected data were analyzed using the Statistical Package for Social Science (SPSS) version 15.

\section{Results}

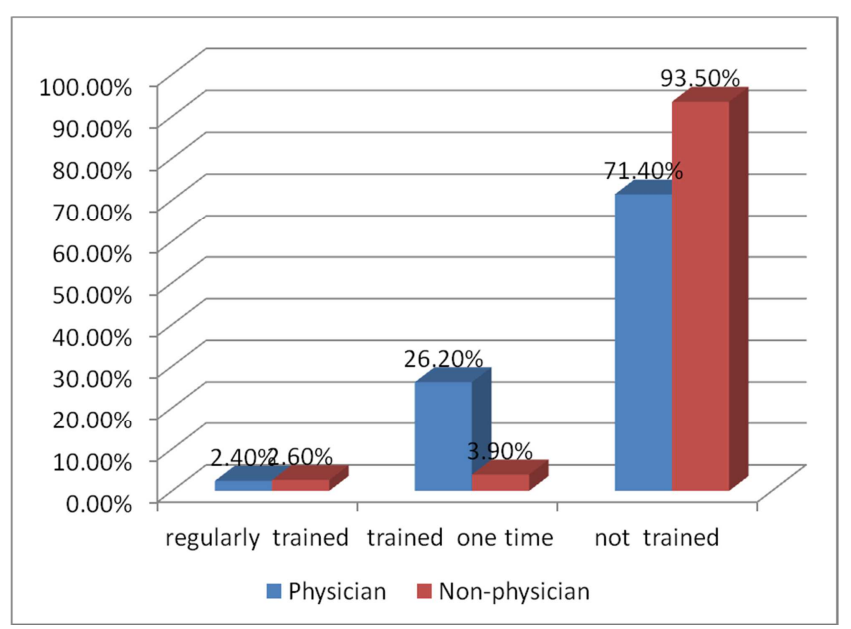

Figure 1. Frequency of in-service training among health care providers. 


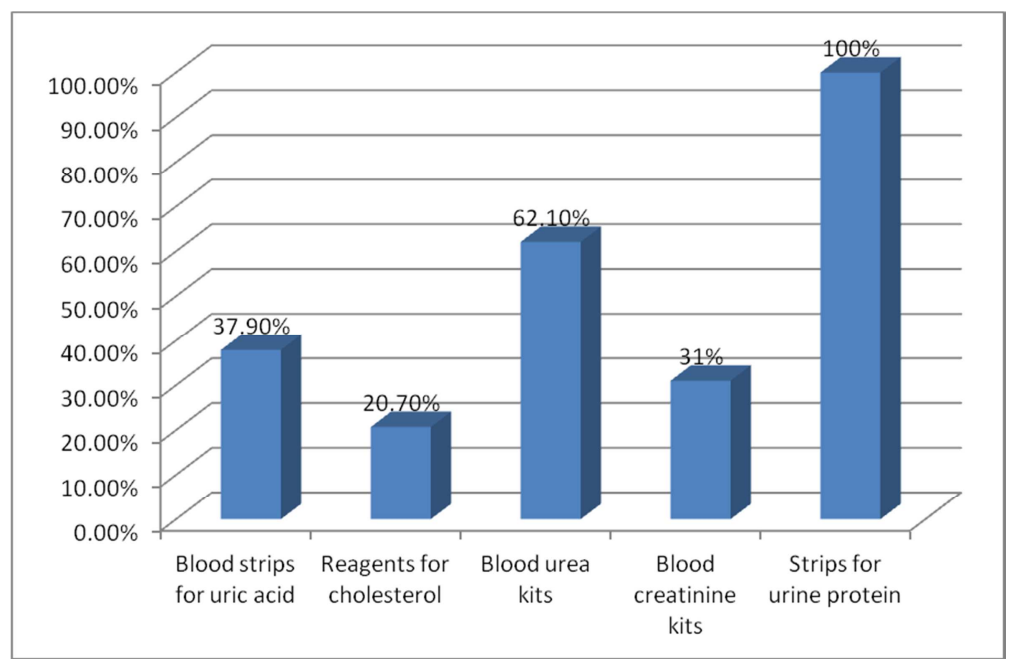

Figure 2. The availability of the laboratory investigations.

Table 1. The outpatient resources.

\begin{tabular}{llllll}
\hline \multirow{2}{*}{ Item } & Seen & & Not seen & \\
\cline { 2 - 6 } & No & Percentage & No & Percentage & Total \\
\hline Examination couch & 29 & 100 & 0 & 0 & 29 \\
Hypertension follow up cards & 11 & 37.9 & 18 & 62.1 & 29 \\
Hypertension or outpatient register & 17 & 58.6 & 12 & 41.4 & 29 \\
Hypertension IEC materials & 1 & 3.4 & 28 & 96.9 & 29 \\
Referral register & 27 & 93.1 & 2 & 6.9 & 29 \\
Hypertension guideline & 3 & 10.3 & 26 & 89.7 & 29 \\
Ambulance & 4 & 13.8 & 25 & 86.2 & 29 \\
Sphygmomanometer & 28 & 96.6 & 1 & 3.4 & 0 \\
Stethoscope & 29 & 100 & 0 & 0 & 0 \\
Adult weighing scale & 27 & 93.1 & 0 & 3.4 & 2 \\
Ophthalmoscope & 7 & 24.2 & 1 & 3.4 & 21 \\
X-ray machine & 6 & 20.7 & 1 & 3.4 & 22 \\
ECG machine & 5 & 17.2 & 1 & 3.4 \\
\hline
\end{tabular}

Table 2. The availability of anti-hypertensive drugs \& the list of essential anti-hypertension drugs.

\begin{tabular}{|c|c|c|c|c|c|}
\hline \multirow{2}{*}{ ITEM } & \multicolumn{2}{|c|}{ SEEN } & \multicolumn{2}{|c|}{ NOT SEEN } & \multirow{2}{*}{ TOTAL } \\
\hline & NO & $\%$ & NO & $\%$ & \\
\hline Aspirin & 25 & 86.2 & 4 & 13.8 & 29 \\
\hline Atenolol & 15 & 51.7 & 14 & 48.3 & 29 \\
\hline Angiotensin Converting Enzyme Inhibitors (Captopril, Enalapril) & 5 & 17.2 & 24 & 82.8 & 29 \\
\hline Furosemide & 18 & 62.1 & 11 & 37.9 & 29 \\
\hline Hydrochlorothiazide & 9 & 31 & 20 & 69 & 29 \\
\hline Isosorbide dinitrate & 4 & 13.8 & 25 & 86.2 & 29 \\
\hline Statins (lovastatin or Simvastatin) & 9 & 31 & 20 & 69 & 29 \\
\hline Calcium channel blockers(Nifidipine retard, amlodipine) & 5 & 17.2 & 24 & 82.8 & 29 \\
\hline Glyceryl trinitrate & 1 & 3.4 & 28 & 96.6 & 29 \\
\hline Adrenergic receptors antagonists (Prazocin) & 3 & 10.3 & 26 & 89.7 & 29 \\
\hline Central a-agonists methyldopa & 9 & 31 & 20 & 69 & 29 \\
\hline Angiotensin II receptor blockers (Losartan) & 4 & 13.8 & 25 & 86.2 & 29 \\
\hline K. sparing diuretics Spironolactone & 3 & 10.3 & 26 & 89.7 & 29 \\
\hline The list of essential anti-hypertension drugs & 15 & 51.7 & 14 & 48.3 & 29 \\
\hline
\end{tabular}

\section{Discussion}

In this study, the currently employed physicians (general practitioners and consultants) were 42 , distributed in all the targeted health facilities (26 HCs and 3 rural hospitals). The physician per population ratio was only 0.5 physicians per 10,000 Populations (the total number of the population of the study area was 868,147$)$. Globally the standard accepted physicians per 10,000 Populations were 14 . However, Sudan is considered one of the least countries regarding the number of physicians per 10,000 Populations (three physicians per 10,000 Populations) (12). Other health care providers were 77 , with different categories: 12 (15.6\%) of them were medical assistants, $66.2 \%$ certified nurses, 4 (5.2\%) nurse-midwives and $10(13 \%)$ health visitors. The nurse per population ratio was found to be only 0.6 per 10,000 populations. The international nursepopulation ratio per 10,000 was found to be; in least developed 
countries were $=2.2$ per 10,000 populations, in other developed countries were $=9.6$ per 10,000 populations, in developed countries were $=74.2$ per 10,000 populations (13). Therefore, the nurse-population ratio was less than the least developed countries. It is clear that, the physicians and nurses per population ratios in the study area are indicative of marked shortages and poor staffing of the health facilities. Training is one of the quality's indicators, in this study as figure 1 illustrated; $71.4 \%$ of the physicians, and $93.5 \%$ of other care providers were not trained. This means that the in-service training on hypertension management is limited, and this can affect the competency of the health care providers, and consequently the quality of services provided to hypertensive patients.

As represented in table 1:

Ambulance was not available in $86.2 \%$ of the health facilities, ambulance is important for timely referral of acutely ill cases; its availability can help much in saving many patients' lives. Hypertension guidelines are useful tools for clinical decision-making; they used to reduce practice variations, guide appropriateness, and measure quality of care (14). In this study, hypertension guidelines were not available in $89.7 \%$ of the health facilities, and the available ones were not national guidelines. Patient records are important components of long-term care for a chronic longstanding disease as hypertension, in this study, records and registries were not available in all health facilities; The recording system was not yet established at the PHC facilities in the study area. Other formats needed for hypertensive patients e.g. patient's follow-up cards and hypertension IEC materials were available only in $37.9 \%$ and 3.45 of health facilities respectively. Standard referral system with all its components including; Booking date and time, regular feedback, follow up of the referred patient, and standard referral registry, did not exist in all the targeted health facilities.

The availability of the equipments in the targeted health facilities; Functioning adult weighing scales, were encountered in $93.1 \%$ of the study health facilities; Functioning sphygmomanometers were seen in $96.6 \%$, of the health facilities, but in all health facilities, there was only one sphygmomanometer, which is inadequate to provide services in any busy health center.

Figure 2 represented the availability of important investigations needed for hypertensive patients'; Test strips for urine albumin available in (100\%), reagent for blood urea available in $62 \%$, strips for uric acid available in nearly $38 \%$, cholesterol available in $(20.7 \%)$, blood creatinine kits available in $(31 \%)$, of the health facilities.

Table 2 illustrated only three out of 13 drugs - and are included in the national drugs list of primary health care level - were available in $86 \%, 62 \%, 52 \%$ of health facilities, and these are; Aspirin, Furosemide and Atenolol respectively, other drugs were available in only some of the health facilities. This means that the patients were obliged to purchase these drugs form private pharmacies (out-pocket price), with all its physical and financial loads.

However, due to limited public sector budget, only a few of the basic diagnostic tests and essential medicines were available at PHC facilities in continuous manner, and people had to spend out of pocket for most of investigations and medicines.

\section{Conclusions}

There was a marked shortage in health care providers, only 0.5 -physician per 10,000 populations, and 0.6 nurses per 10,000 population. Other resources were inadequate to provide quality services for hypertensive patients at primary health care level

\section{References}

[1] Regional Office of East Mediterranean World Health Organization, Regional Office for Eastern Mediterranean, Clinical guideline for management of hypertension. EMRO technical publication Cairo 2005.

[2] Federal Ministry of Health, SHHS 2010 chronic diseases.

[3] Federal Ministry of Health, Annual health statistical report 2008.

[4] Chobanian AV et al. Seventh report of the Joint National Committee on prevention, detection, evaluation and treatment of high blood pressure. Hypertension, 2003, 42: 1206-1252.

[5] WHO Preventing chronic disease: a vital investment. Geneva, World Health Organization, 2005.

[6] The World Health Report 2002: reducing risks, promoting healthy life. Geneva, World Health Organization, 2002.

[7] Leeder $\mathrm{S}$ et al. A race against time: the challenge of cardiovascular disease in developing economies. New York, the Center for Global Health and Economic Development, 2004.

[8] WHO Secondary prevention of non-communicable diseases in low- and middle-income countries through community-based and health service interventions. Report of the Cambridge Meeting. World Health Organization and Well comeTrust, 2001.

[9] WHO Cardiovascular disease prevention. Translating evidence into action. Geneva, World Health Organization, 2005.

[10] WHO CVD risk management package for low-and mediumresource settings. Geneva, World Health Organization, 2002.

[11] WHO Global strategy for the prevention and control of noncommunicable diseases. Report by the Director General. Geneva, World Health Organization, 2000 (Document A53/14).

[12] World Health Statistics 2012.

[13] K. PARK, M. B. B. S., M. S., textbook of preventive and social medicine, eighteenth edition; M/s Banarsidas Bhanot; Publishers; 1167, Prem Nagar, Jabalpur, 482001 (India), page 26, (able 4 selected health and socio-economic indicators.

[14] Bodenheimer T, Wagner EH, Grumbach K. Improving primary care for patients with chronic illness: the chronic care model, part 2. JAMA. 2002; 288: 1909-1914. 